\title{
Preparing for a trial to test a postpartum weight retention intervention among low income women: feasibility of a protocol in a community-based organization
}

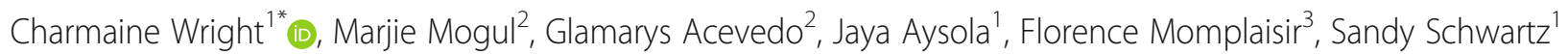
and Judy Shea'

\begin{abstract}
Background: Postpartum weight retention (PPWR) causes intergenerational harm, negatively affecting a mother's cardiovascular health and ability to have future healthy pregnancies. Low-income minority women are at highest risk for PPWR with little guidance concerning timeline or strategy to lose weight after delivery. An academic-community partnership conducted observational and focus group work to develop an intervention for PPWR among low-income mothers. This study's objective is to determine the feasibility of implementing a PPWR intervention trial in partnership with a community-based organization (CBO) serving low-income families with social service support.
\end{abstract}

Methods: We analyzed five implementation outcomes in this feasibility study: acceptability, adoption, appropriateness, penetration, and sustainability. Other secondary outcomes were the change in psychosocial and clinical outcomes from baseline to one year following the intervention delivery.

Results: An academic-community partnership developed and piloted a postpartum weight retention intervention among 17 participants that included 1) six weeks of interactive daily health texting, 2) exercise assistance with baby carrier, home exercise program, and pedometer provision, 3) two live healthy eating and baby feeding workshops, and 4) two 45-min home visits over one year to provide social support and acquire followup data. Implementation outcomes demonstrate an intervention supported by the organization and accepted by end-users, with increased capacity of the $\mathrm{CBO}$ to test and deliver an effective intervention. Weight loss was achieved by the majority of participants at one year (Md -5 pounds (IQR $=-14.5-0.3)$.

Conclusion: We made protocol enhancements to the developed intervention based on the analysis of this study, and now prepare for a funded randomized controlled trial $(\mathrm{RCT})$ in a community-based setting. Our central hypothesis is that low-income women who participate in a multi-component, low cost-intervention delivered by a CBO will have less postpartum weight retention than those women who do not participate in the program.

Trial registration: The trial was retrospectively registered, ID NCT02867631, 8/11/16.

\footnotetext{
* Correspondence: smich@mail.med.upenn.edu

'Division of General Internal Medicine, Blockley Hall, 423 Guardian Dr,

Philadelphia, PA 19104, USA

Full list of author information is available at the end of the article
} 


\section{Background}

Postpartum weight retention (PPWR) is associated with future unhealthy pregnancies, long-term obesity, and chronic cardiovascular disease [1-4]. Despite the growing awareness of the dangers of postpartum weight retention, neither an ideal time nor method to return to pre-pregnancy weight has been established in the literature [4-8]. Of additional concern is the recent demonstration of the postpartum period as a life stage characterized not only by weight retention, but also by susceptibility to excessive weight gain $[9,10]$. Though Hispanic and African-American low-income mothers are at greatest risk for PPWR, postpartum gain, and adverse future birth outcomes, it remains a poorly-understood phenomenon. PPWR is thought to be the result of a variety of environmental, socioeconomic, and educational factors including starting pregnancy at unhealthy weight, gaining too much weight during pregnancy, maternal stress, and lack of breastfeeding and knowledge about healthy behaviors [11-14].

In the literature, several postpartum weight retention interventions have been studied. Intervention delivery was undertaken by a range of health professionals in various settings, but no definitive pattern was seen in successful interventions, and few took place in community-based settings [15-17]. Phelan et al. recruited low-income mothers from Women Infant and Children (WIC) Supplemental Nutrition Program offices and was the first to implement an internet-based treatment program with monthly group sessions $[18,19]$. Van der Pligt et al. implemented a largely online intervention with dietician counseling by phone and calorie tracking by app among first time mothers in Australia, and also encouraged the use of less burdensome interventions that do not require face-to-face contact among busy new mothers [20]. Fernandez et al. implemented a trial testing an electronically-mediated behavioral intervention in an urban population of mothers recruited from an obstetric practice [21]. All these studies required the use of a smart phone or computer to participate.

In addition to focusing on socioeconomically disadvantaged mothers [22-24], racial and ethnically-sensitive approaches among Hispanic and African-American mothers have been piloted [20, 22, 23, 25-30]. ChasanTaber et al. focused on Hispanic mothers in Massachusetts in an effort to test a culturally and linguistically modified intervention utilizing multimodal sources of counseling and goal setting for new mothers [22]. With efforts to prevent postpartum weight retention in African American mothers meeting multiple challenges, Herring et al. effectively recruited African American Medicaid recipients to a positive intervention study that included Facebook support and health coaching provided by telephone, with very little loss to followup at six months [28]. Though it seems clear that diet alone or a combination of diet and exercise will allow mothers to achieve postpartum weight loss as demonstrated by Cochrane Review [31], it remains unclear as to the optimal setting, delivery method, method of contact, or intervention length. As low-income mothers often have little health care beyond a recommended six week postdelivery appointment [24], this study will provide some clarity on how we might provide longer-term postpartum care for women in community based settings.

As part of a larger research project to reduce postpartum weight retention by determining the effectiveness of a community-based intervention among low income mothers, the University of Pennsylvania research team partnered with a community-based organization providing social services to low income women with a home visitation model implemented by community health workers (CHWs). This partnership performed observational [13] and focus group work (in press) leading to an holistic intervention informed by the tenets of community-engaged research (CER) and the theoretical framework of Ajzen's Theory of Planned Behavior (TPB) $[32,33]$. Specifically, we aimed to improve participants' perceived behavior control (self-efficacy), subjective norms, and attitudes regarding postpartum weight management by developing four intervention components: 1 ) Motivational appeals to augment self-efficacy delivered by daily health texting for a period of six weeks; 2) Provision of environmental aids to improve self-efficacy for exercise and attitudes towards exercise by encouraging daily at-home physical activity with a twentyminute baby carrier exercise program and continuous pedometer usage; 3 ) Task-oriented support to improve knowledge and attitudes for healthy eating and infant feeding through two live ninety-minute classes; and 4) Provision of peer support to combat perinatal depression, influence subjective norms, and enhance perception of social support with class and two forty- fiveminute home visits over one year following delivery. The curriculum for the task-oriented support was delivered during the two classes and health texting. It directly answered the call for help we demonstrated with focus group work among staff and clients of the $\mathrm{CBO}$. In regards to postpartum health, women asked for structure, social support, assurance they would not injure themselves or their babies, and time saving strategies to eat and exercise in a healthy manner after delivery.

The curricula therefore included meal planning, avoidance of sugar-sweetened beverages, and actionable healthy texts that include 100-cal snack ideas. The healthy feeding curricula delivered task-oriented instruction focused on lactation support provided in class and 
by phone if requested, bottling education, and goals of infant solid food introduction. The addition of the infant feeding instruction was prompted by focus group work demonstrating many client's requests to know more about ways to combat pediatric obesity and its intergenerational transmission [14, 34].

The current study focuses on determining the feasibility of implementing a randomized clinical trial of these intervention components among clients of the partnering community-based organization, primarily focusing on five out of eight implementation outcomes defined by Proctor et al. including acceptability, adoption, appropriateness, penetration, and sustainability [35]. Since we were adjusting protocol in a dynamic fashion to maximize feasibility, we did not test fidelity or cost in this study.

\section{Methods}

\section{Overview}

The intervention components are informed by the Theory of Planned Behavior as described. The academiccommunity partnership developed the overall study design, and chose the outcomes based on a literature review of the behaviors we aimed to change: increase in meal planning, increase in eating fruits and vegetables, decrease in sugar-sweetened beverages, decrease in feelings of social isolation, increase in walking and incorporating the baby into exercise, and increase in breastfeeding time. The primary outcome is therefore postpartum weight retention (calculated as an absolute difference in pounds between one year postpartum and initial study weight obtained at 6-12 weeks postpartum). The secondary outcomes will be improved dietary composition (by dietary recall, from our validated nutrition knowledge and behavior psychosocial constructs [13]), decreased risk of depression (by Edinburgh screen [36]), improved breastfeeding time (in weeks) and maintained infant growth (weight for length).

\section{Participants}

The CHWs were engaged at staff meetings and through email, and encouraged to refer their pregnant and postpartum clients to the study team for a feasibility study of the intervention. The target population was a convenience sample of low-income postpartum clients of the $\mathrm{CBO}, 100 \%$ of which are WIC-eligible. Inclusion criteria included English or Spanish-speaking recently postpartum non-pregnant participant, with self-reported prepregnancy $B M I \geq 18.5 \mathrm{~kg} / \mathrm{m}^{2}$, delivery of single, live term (>37 weeks gestation) infant, and cell phone with text messaging capabilities (92\% clients in preliminary work). Women were excluded from the pilot study if their prepregnancy BMI was $<18.5 \mathrm{~kg} / \mathrm{m}^{2}$ (underweight). Additionally, we terminated a mother from the study if BMI was not maintained above $18.5 \mathrm{~kg} / \mathrm{m}^{2}$, or infant weight loss was noted at any of the weigh-ins, with referral to her healthcare provider. Our goal was to recruit 15 people which represented $10 \%$ of our initial proposed sample size needed for the RCT $(n=150)$, to detect a difference of two pounds of weight between groups with a standard deviation of four.

\section{Conduct of pilot intervention}

Over three months, 26 women were referred of which 17 were eligible and in the window of 6-12 weeks postpartum at the time of the first study session. This window was chosen to engage women in the intervention after attending their recommended six week postpartum follow up visit with their health care provider to ensure safety to exercise. Women were recruited from 3 of the $\mathrm{CBO}$ sites and attended an initial ninety-minute class at their neighborhood site. They returned for a follow up class six weeks later, with 45-min home visits at six and twelve months post-delivery. Cab rides to attend classes were provided, as well as childcare for older children, and an invitation for a partner or support person to accompany the participant. The most intensive part of the intervention was between the two classes during which time women were encouraged to use the baby carrier with associated 20-min exercise program and pedometer while receiving and answering forty two days of text messages. The timeline for receipt of these intervention components is noted in Table 1.

\section{Measures}

1) Acceptability of the intervention trial was defined in a few ways: 1) rate of initial enrollment of those referred; 2) rate of attrition over the one year followup period; 3) engagement with the environmental aids given (time spent using the baby carrier, exercise program, and pedometers and rate of texting back study data); 4) post intervention self-reported utilization of environmental aids; and 5) post intervention reported satisfaction; and 6) positive attitudes towards planned randomization.

2) Adoption was defined qualitatively by the $\mathrm{CBO}$ leadership's support of the study as demonstrated by the internal and external communication of the intervention and organizational development around the study.

3) Appropriateness was defined qualitatively by the academic-community partnership as the ability to recruit and retain participants at a rate comparable to other community-based programs, with the use of one research assistant, an interventionist, and the current geographic resources. 
Table 1 Intervention Components

\begin{tabular}{|c|c|c|c|c|}
\hline Event & TO & T6 weeks & T6 months & T12 months \\
\hline Class: Healthy Eating and Feeding & $x$ & $x$ & & \\
\hline Receipt of home exercise aids: pedometer, baby carrier, and home exercise program booklet & $x$ & & & \\
\hline Daily Motivational Texting and self-report of pedometer steps and time spent using baby carrier & $x$ & $x$ & & \\
\hline Home Visits & & & $x$ & $x$ \\
\hline Vitals and Assessment & $x$ & $x$ & $x$ & $x$ \\
\hline
\end{tabular}

4) Good penetration was defined as the widespread referral of clients from the eight geographic sites of the $\mathrm{CBO}$.

5) Sustainability was defined as the ability to acquire grant funding for a larger RCT and the ability of the $\mathrm{CBO}$ to assume all administrative and logistical components of the intervention with minimal outside assistance.

6) Nutrition knowledge and psychosocial factors were evaluated by a comprehensive tool previously validated by the academic-community partnership [13], and was measured at baseline, six weeks, six months, and twelve months.

7) Vital sign assessment including maternal and child height and weight was measured at baseline, six weeks, six months, and twelve months.

\section{Data collection}

A CHW and research assistant collected paper surveys at each class, and at six and twelve months postpartum. A script was written for both the classes and home visits to ensure fidelity of the delivered intervention. Anthropometric training was provided to the research assistant using the National Health and Nutrition Examination Survey videos and protocol to ensure the collection of research-standard measurements, including maternal weight on an electronic scale measured to the nearest $0.1 \mathrm{~kg}$ (Seca Robusta 213), and height measured by a portable stadiometer (Seca 213). The infant weight was acquired by measuring both mother and baby on the scale. Infant length was acquired using the Measure Mat II Infantometer (Hopkins Medical). Participants reported their pre-pregnancy weight and gestational weight gain. All survey data were entered into REDCap (R6.16.4) for later analysis by the Stata statistical package (STATA 13, college Station TX).

\section{Data analysis}

Univariate analysis was completed with chi-squared (categorical variables) and $\mathrm{t}$-tests (continuous variables) to determine differences among those participants who were retained and those who dropped out. Mean and median postpartum weight change was calculated with interquartile ranges.

\section{Results}

Acceptability and penetration: During the intervention pilot, we focused on operations and protocol enhancement by looking at the implementation outcomes described. Initially, we enrolled 17 women, with 5/17 (30\%) attrition by six weeks, $6 / 17$ (35\%) at 6 months, and 8/17 $(47 \%)$ attrition at one year. For this initial pilot recruitment, we relied on referrals from community health workers, with disparate rates of referral across the neighborhood sites. For example all women came from 3 sites where the CHWs had a previous interest in health and nutrition and had an increased level of success with recruitment. 5-8 women were referred by these advocates monthly, and demographics mirrored those of the population the $\mathrm{CBO}$ serves and those studied in focus group work. At the time, 3 other sites were being renovated and the CHW's were busy managing the transition beyond their normal caseload and didn't refer any clients from these sites. Yet another site had an ongoing transition of leadership and did not refer any clients during the study period. In terms of engagement by the mothers, during the intensive portion of the intervention, 8/12 (66\%) reported use of the baby carrier more than half the days of the week; $9 / 12$ (75\%) reported pedometer use more than half the days of the week. Of those followed through six months, 8/11 (73\%) continued to use the baby carrier and pedometer provided at least once a week, and 7/11 (64\%) viewed the packet of exercise, eating, and baby feeding tips at least once a week. Engagement with texting back footsteps and baby carrier time was variable, with $9 / 11$ (82\%) of women texting at least 3 times a week. $100 \%$ said they were satisfied and would tell a friend about the program. Those who dropped out of the study by one year were similar to those retained in regards to starting body mass index (BMI), self-efficacy, perinatal depression risk, racial/ethnic background, and days spent returning texts. Those who dropped out did have more children living at home as an important difference (Table 2). When asked about randomization, 9/11 (82\%) of participants said they would be willing to be randomized as long as they still received the baby carrier and handout information.

Adoption, Appropriateness, and Sustainability: Organizational support by the $\mathrm{CBO}$ was strong. The study was featured on the CBO's website, and in the 
Table 2 Pilot Study Participant Characteristics

\begin{tabular}{lll}
\hline & Population 1 year post-delivery $(n=9)$ & Drop out population $(n=8)$ \\
\hline Initial age & $25.6(4.6)$ years & $25.4(4.1)$ years \\
Race/Ethnicity: & $78 \%$ & $62.5 \%$ \\
Black & $12.5 \%$ & $25 \%$ \\
Hispanic & $12.5 \%$ & $12.5 \%$ \\
$\quad$ White & $30.7(4.0) \mathrm{kg} / \mathrm{m}^{2}$ & $31.9(4.1) \mathrm{kg} / \mathrm{m}^{2}$ \\
Initial Body Mass Index (BMI) & $38.7(3.8)$ inches & $38.5(4.0) \mathrm{inches}$ \\
Initial waist circumference & $89 \%$ & $88 \%$ \\
WIC recipient & 1.2 & 3.2 \\
Number children at home & 14 & 15 \\
Days of return texting &
\end{tabular}

annual report. A story from one participant was featured in a CBO-wide email and blog. We were given conference room space and use of the fleet of $\mathrm{CHW}$ vehicles to acquire study tools and transportation to study sessions at no charge. We were successful in acquiring a pilot grant from the University of Pennsylvania Implementation Science Working Group, and a larger foundation grant from the Aetna Foundation to fund a CHW turned part -time research assistant who was based at the $\mathrm{CBO}$ but met with the PI at the University of Pennsylvania once weekly thus providing a critical link in the partnership, increasing $\mathrm{CBO}$ capacity. We employed a local lactation consultant and interventionist. During the follow up phase, we were able to secure additional funds in the form of further foundation grants, one of which emanated from the $\mathrm{CBO}$ itself which allowed us to plan for an RCT of the intervention using the same staffing.

Psychosocial and weight assessment: Mean selfefficacy for healthy behaviors improved over the course of the six week intensive intervention for the $12 / 17$ who were retained at six weeks(from 6.9 (2.7) to 5.2(2.6), $p=$ $0.04)$. However, self-efficacy returned to baseline by six and twelve months without significant change. Of those retained, more than half $(7 / 12,58 \%)$ lost weight over the course of the six week intensive intervention (Md -1.0 pounds (IQR $=-4.1-1.8)$ ), with 7/9 (78\%) losing at one year post-delivery $(\mathrm{Md}-5$ pounds $(\mathrm{IQR}=-14.5-0.3)$ ). $25 \%$ of women were at risk for perinatal depression at the start of the program, and this point prevalence remained stable over time.

\section{Discussion}

Based on the results of the pilot intervention, we made key protocol changes to effectively deliver the components during RCT testing. For example, during the pilot, we relied on CHW referrals to generate our study subjects. However, they had a large number of responsibilities they accomplish for the $\mathrm{CBO}$ home visitation program, and recruitment was subject to the environment at the neighborhood site. Recruitment for our study was limited by the amount of time the CHWs had, renovation or leadership changes at the neighborhood site, and the severity of an individual community health worker's case load, as well as personal interest in health and nutrition. Since the level of penetration and attrition during the pilot was unacceptable to us and more than the $40 \%$ often encountered by community-based intervention studies, for our RCT intervention study, we will instead use a database of all postpartum clients managed by the $\mathrm{CBO}$ to recruit our participants, with $\mathrm{CHWs}$ providing input about safety information or mental health status regarding the participant. This change will allow us to contact every postpartum client as potential study subjects, and will allow the CHWs to focus on their primary responsibility of direct service provision. We can also ensure better penetration by contacting pregnant women from all geographic sites. We now know the importance of budgeting future funding to allow certain CHWs to be designated wellness champions with a reduced case load to allow for research study participation and program implementation, ensuring sustainability of the program once implemented. We will improve our emergency contact procedures for depressed participants as we found such a high prevalence during the pilot, and we will incentivize engagement with the intervention by providing an extra gift card to the woman who texts back the most in her cohort.

We also decided to increase the dose of the intensive intervention by lengthening class time to $120 \mathrm{~min}$ and home visit education to $60 \mathrm{~min}$ as we saw a significant initial change in self efficacy which returned to baseline at six and twelve month follow-up. The weight outcomes at six and twelve months post-delivery helped us to determine effect size and plan for our sample size in the RCT. To detect a 4 pound difference in weight change at one year post delivery using the standard deviations acquired (8), we would need a control population of 85 
and an intervention population of 85 (an increase in sample size over our originally planned sample size). Attrition rates also aided in planning for our proposed recruitment sample. We used several previously described strategies for retention of our subjects in this community setting and anticipate the attrition seen in other community-based interventions (no more than 40\%) [37]. We employ CBO staff as research study staff and thought critically about the type and timing of incentives to aid in recruitment. We piloted multiple procedures for participant follow-up during the pilot, including collecting emergency contact information at the outset, maintaining contact over the course of the entire year by text messages, and incentivizing updating contact information. The use of all three brought about the highest level of retention in our final cohort of women.

\section{Conclusion}

This pilot study was a key step in planning for an RCT to study efficacy and cost-effectiveness of the holistic intervention, thus deepening the knowledge of best practices that exists for the combat of postpartum weight retention and its intergenerational downstream effects. At every point, community insight and collaboration has been considered. We were encouraged by this feasibility study that serves as a proof of concept and readies us for further testing with improved implementation outcomes and sample size to detect differences among control and intervention groups. Additionally, in this small group of women, we were able to assist women in decreasing their weight in the postpartum period while increasing their self-efficacy, thus improving their personal and familial health.

\section{Abbreviations}

BMI: Body mass index; CBO: Community-based organization;

CER: Community-engaged research; CHWs: Community health workers; PPWR: Postpartum weight retention; RCT: Randomized controlled trial; TPB: Theory of Planned Behavior

\section{Acknowledgements \\ Special thanks to Maternity Care Coalition, without whose partnership, this work would not be possible.}

\section{Funding}

This study was funded by the University of Pennsylvania, Aetna Foundation, and the Robert Wood Johnson Foundation. All three funding sources supported the biostatistical assistance, intervention supplies, and staffing necessary for study completion and were acquired during the same time period. The Aetna Foundation award additionally supported cab rides for participants, gift cards for participant incentives, and STAT software. The collection, analysis, and interpretation of the results was supported by all three sources as well.

\section{Availability of data and materials}

The datasets used during and/or analyzed during the current study are available from the corresponding author on reasonable request.

\section{Authors' contributions}

GA, CW, MM, SS and JS participated in the design, implementation, data collection, analysis, and interpretation of the study results. FM and JA provided design and content expertise and edited the manuscript. CW, JS, and SS performed the statistical analysis and preparation of the methods section. JA provided feedback on the statistical analysis and assisted in preparing the result and discussion sections. CW and MM conceived of the study, participated in its design and coordination and drafted the initial manuscript. All authors approved the final manuscript and accept responsibility for the study.

\section{Ethics approval and consent to participate}

Ethics approval was granted by the University of Pennsylvania Institutional Review Board and written consent to participate was collected from each participant (\#808132).

\section{Consent for publication}

Not applicable.

\section{Competing interests}

The authors declare that they have no competing interests.

\section{Publisher's Note}

Springer Nature remains neutral with regard to jurisdictional claims in published maps and institutional affiliations.

\section{Author details}

'Division of General Internal Medicine, Blockley Hall, 423 Guardian Dr, Philadelphia, PA 19104, USA. ²Maternity Care Coalition, 2000 Hamilton Avenue, Suite 205, Philadelphia, PA 19130, USA. ${ }^{3}$ Drexel School of Medicine, Department of Medicine, Infectious Diseases, Partnership Comprehensive Care Practice, 1427 Vine Street, 2nd Floor, Philadelphia, PA 19102, USA.

Received: 24 July 2017 Accepted: 14 January 2018

Published online: 25 January 2018

\section{References}

1. Castro LC, Avina RL. Maternal obesity and pregnancy outcomes. Curr Opin Obstet Gynecol. 2002;14(6):601-6.

2. Olson CM. A call for intervention in pregnancy to prevent maternal and child obesity. Am J Prev Med. 2007;33(5):435-6.

3. Schieve LA, Cogswell ME, Scanlon KS. Trends in pregnancy weight gain within and outside ranges recommended by the Institute of Medicine in a WIC population. Matern Child Health J. 1998;2(2):111-6.

4. Gore SA, Brown DM, West DS. The role of postpartum weight retention in obesity among women: a review of the evidence. Ann Behav Med. 2003; 26:149-59.

5. Baker JL, Gamborg M, Heitmann BL, Lissner L, Sorensen TIA, Rasmussen KM. Breastfeeding reduces postpartum weight retention. Am J Clin Nutr. 2008; 88(6):1543-51

6. Kinnunen TI, Pasanen M, Aittasalo M, Fogelholm M, Weiderpass E, Luoto R. Reducing postpartum weight retention-a pilot trial in primary health care. Nutr J. 2007:6(21)

7. Leermakers EA, Anglin K, Wing RR. Reducing postpartum weight retention through a correspondence intervention. Int J Obes Relat Metab Disord. 1998;22(11):1103.

8. Polley BA, Wing RR, Sims CJ. Randomized controlled trial to prevent excessive weight gain in pregnant women. Int J Obes Relat Metab Disord. 2002;26(11):1494-502.

9. Taveras E. First steps for mommy and me: a pilot intervention to improve nutrition and physical activity behaviors of postpartum mothers and their infants. Matern Child Health J. 2011:15:1217-27.

10. Lipsky LM, Strawderman MS, Olson CM. Maternal weight change between 1 and 2 years postpartum: the importance of 1 year weight retention. Obesity (Silver Spring). 2012;20(7):1496-502.

11. Krummel DA. Postpartum weight control: a vicious cycle. J Am Diet Assoc. 2007;107(1):37-40.

12. Schauberger CW, Rooney BL, Brimer LM. Factors that influence weight loss in the puerperium. Obstet Gynecol. 1992;79(3):424-9.

13. Wright CS, Mogul M, Shea J. Psychosocial factors associated with gestational weight gain in a low income cohort. JHCPU. 2013;241:332-43.

14. Lipsky LM, Strawderman MS, Olson CM. Weight-related self-efficacy in relation to maternal body weight from early pregnancy to 2 years postpartum. Matern Child Nutr. 2016;12(3):569-78. 
15. Amorim AR, Linne YM, Lourenco PMC. Diet or exercise, or both, for weight reduction in women after childbirth. Cochrane Database Syst Rev. 2007;3: CD005627.

16. Kuhlmann AKS, Dietz PM, Galavotti C, England LJ. Weight-management interventions for pregnant or postpartum women. Am J Prev Med. 2008; 34(6):523-8.

17. van der Pligt P, Willcox J, Hesketh KD, Ball K, Wilkinson S, Crawford D, Campbell K. Systematic review of lifestyle interventions to limit postpartum weight retention: implications for future opportunities to prevent maternal overweight and obesity following childbirth. Obes Rev. 2013;14(10):792-805.

18. Phelan S, Brannen A, Erickson K, Diamond M, Schaffner A, Munoz-Christian K, Stewart A, Sanchez T, Rodriguez VC, Ramos DI, et al. 'Fit moms/mamas Activas' internet-based weight control program with group support to reduce postpartum weight retention in low-income women: study protocol for a randomized controlled trial. Trials. 2015;16(59)

19. Phelan S, Phipps MG, Abrarms B, Darroch F, Grantham K, Schaffner A, Wing RR. Does behavioral intervention in pregnancy reduce postpartum weight retention? Twelve-month outcomes of the fit for delivery randomized trial. Am J Clin Nutr. 2014:99(2):302-11.

20. van der Pligt P, Ball K, Hesketh KD, Teychenne M, Crawford D, Morgan PJ, Collins $\mathrm{CE}$, Campbell KJ. A pilot intervention to reduce postpartum weight retention and central adiposity in first-time mothers: results from the mums OnLiNE (Online, lifestyle, Nutrition \& Exercise) study. J Hum Nutr Diet. 2017; 56:23-38.

21. Fernandez ID, Groth SW, Reschke JE, Graham ML, Strawderman M, Olson CM. eMoms: electronically-mediated weight interventions for pregnant and postpartum women. Study design and baseline characteristics. Contemp Clin Trials. 2015:43:63-74

22. Chasan-Taber L, Marcus BH, Rosal MC, Tucker KL, Hartman SJ, Pekow P, Stanek E 3rd, Braun B, Solomon CG, Manson JE, et al. Proyecto mama: a lifestyle intervention in overweight and obese Hispanic women: a randomised controlled trial-study protocol. BMC Pregnancy Childbirth. 2015;15:157.

23. Phelan S, Hagobian T, Brannen A, Hatley KE, Schaffner A, Munoz-Christian K, Tate DF. Effect of an internet-based program on weight loss for low-income postpartum women: a randomized clinical trial. JAMA. 2017:317(23):2381-91.

24. Gould Rothberg BE, Magriples U, Kershaw TS, Rising SS, Ickovics JR. Gestational weight gain and subsequent postpartum weight loss among young, low-income, ethnic minority women. Am J Obstet Gynecol. 2011; 204(1):52.e51-11.

25. Asci O, Rathfisch G. Effect of lifestyle interventions of pregnant women on their dietary habits, lifestyle behaviors, and weight gain: a randomized controlled trial. J Health Popul Nutr. 2016:35:7.

26. Davis JN, Shearrer GE, Tao W, Hurston SR, Gunderson EP. Dietary variables associated with substantial postpartum weight retention at 1-year among women with GDM pregnancy. BMC Obesity. 2017;4:31.

27. Elliott-Sale KJ, Barnett CT, Sale C. Systematic review of randomised controlled trials on exercise interventions for weight management during pregnancy and up to one year postpartum among normal weight, overweight and obese women. Pregnancy hypertension. 2014;4(3):234.

28. Herring SJ, Cruice JF, Bennett GG, Darden N, Wallen JJ, Rose MZ, Davey A, Foster GD. Intervening during and after pregnancy to prevent weight retention among African American women. Prev Med Rep. 2017;7:119-23.

29. Spencer L, Rollo M, Hauck Y, MacDonald-Wicks L, Wood L, Hutchesson M, Giglia R, Smith R, Collins C. The effect of weight management interventions that include a diet component on weight-related outcomes in pregnant and postpartum women: a systematic review protocol. JBI Database System Rev Implement Rep. 2015;13(1):88-98.

30. van der Pligt P, Ball K, Hesketh KD, Crawford D, Teychenne M, Campbell K. The views of first time mothers completing an intervention to reduce postpartum weight retention: a qualitative evaluation of the mums OnLiNE study. Midwifery. 2017(56):23-8.

31. Amorim Adegboye AR, Linne YM. Diet or exercise, or both, for weight reduction in women after childbirth. Cochrane Database Syst Rev. 2013;(7): Cd005627.

32. Ajzen I. From intentions to actions: a theory of planned behavior. In: Kuhi J, Beckmann J, editors. Action and control: From cognition to behavior. Heidelberg: Springer; 1985. p. 11-39.

33. Ajzen I, Madden TJ. Prediction of goal-directed behavior: Attitudes, intentions, and perceived behavioral control. J Exp Soc Psychol. 1986; 22:45374.
34. Olson CM, Baker IR, Demment MM, Graham ML, May JJ, Strawderman MS, Wells NM. The healthy start partnership: an approach to obesity prevention in young families. Family Commun Health. 2014;37(1):74-85.

35. Proctor $E$, Silmere $H$, Raghavan $R$, Hovmand $P$, Aarons G, Bunger A, Griffey R, Hensley M. Outcomes for implementation research: conceptual distinctions, measurement challenges, and research agenda. Admin Pol Ment Health. 38(2):65-76.

36. Boyd RC, Le HN, Somberg R. Review of screening instruments for postpartum depression. Archives of Women's Mental Health. 2005;8(3): 141-53.

37. Flores G. Devising, implementing, and evaluating interventions to eliminate health care disparities in minority children. Pediatrics. 2009; 124(Supp| 3):S214-23.

\section{Submit your next manuscript to BioMed Central and we will help you at every step:}

- We accept pre-submission inquiries

- Our selector tool helps you to find the most relevant journal

- We provide round the clock customer support

- Convenient online submission

- Thorough peer review

- Inclusion in PubMed and all major indexing services

- Maximum visibility for your research

Submit your manuscript at www.biomedcentral.com/submit
Biomed Central 